\title{
CT Patterns Among Covid-19 Patients During the Second Wave: A Single Institute Study
}

\author{
Aya Yassin ${ }^{1}$, Maryam A. Abdelkader ${ }^{2}$, Rehab M. Mohammed ${ }^{2}$, Mona A. Nagi ${ }^{* 1}$
}

Departments of ${ }^{1}$ Radiology and ${ }^{2}$ Chest Diseases, Faculty of Medicine, Ain Shams University, Cairo, Egypt

*Corresponding author: Mona Ali Mohamed Ali Nagi, Mobile: +201013367332, Email: mona.babydoc@yahoo.com

\begin{abstract}
Background: The COVID-19 pandemic caused a worldwide dramatic loss of human life. The second wave of coronavirus showed a very rapid spread. Objective: This study aimed to retrospectively analyze the laboratory findings and chest computed tomography (CT) features in patients with suspected coronavirus disease (COVID-19) pneumonia during the second wave. Patients and methods: From November 2020 to February 2021, a total of 295 patients were admitted to our hospital with suspected COVID-19 pneumonia. They underwent multiple laboratory tests including (s. ferritin, CBC) as well as non-contrast CT. Only 144 patients had PCR results available. The CT findings were reported as regards the presence of ground glassing, consolidation and pleural effusion. Results: 198 (67.1\%) showed ground glassing and 36.9\% (109 cases) had consolidation in their CT. These lesions were bilateral in 181 cases about $63.3 \%$ being single in in 71 cases $(25.3 \%)$. Pleural effusion was found in 202 cases $(68.7 \%)$. Significant correlation was found between CRP, s. ferritin and d-dimer with presence of consolidation. Significant decreased neutrophil count and decreased DD among positive in comparison with negative ground glassing. PCR results were available in only 144 out of 295 patients (48.8\%). It was positive in 100 out of 144 patients (69.4\%) and negative in 44 patients (30.6\%). Highly significant relation between COVID-19 Reporting and Data System (CORADS) categories \& PCR among all studied cases. Conclusion: During the second wave, the CT findings were similar to wave one with PCR proved COVID-19 cases more frequent with higher CO-RADS and RSNA categories.
\end{abstract}

Keywords: COVID-19, Pneumonia, Computed Tomography.

\section{INTRODUCTION}

The second wave of coronavirus disease (COVID-19) has caused a major worldwide dramatic increase in COVID-19 cases with increase in the death rates globally. Chest imaging is important in assessment of patients with COVID-19 ${ }^{(\mathbf{1})}$. The chest imaging findings of COVID-19 were first described in January 2020, namely bilateral ground-glass opacities in most of the hospitalized patients ${ }^{(2)}$. Several studies were done to evaluate the diagnostic value of chest CT in COVID-19 cases. CT reporting systems for COVID-19 have evolved, namely the COVID-19 Reporting and Data System (CO-RADS) ${ }^{(3)}$ as well as the Radiological Society of North America (RSNA) classification system for reporting COVID-19 pneumonia ${ }^{(4)}$. CO-RADS includes five categories (CO-RADS 1 to 5) as shown in table (1). The employment of these standardized diagnostic classification systems decreases observer variation and improve the clinical communication ${ }^{(5)}$.

Table (1): CORADS classification for COVID-19 pneumonia (The radiology assistant)

\begin{tabular}{|l|l|l|}
\hline \multicolumn{2}{|c|}{ CO-RADS (Level of suspicion COVID-19 infection) } \\
\hline CO-RADS 1 & No & $\begin{array}{l}\text { CT findings } \\
\text { abnormalities }\end{array}$ \\
\hline CO-RADS 2 & Low & $\begin{array}{l}\text { Abnormalities consistent with } \\
\text { infections other than COVID-19 }\end{array}$ \\
\hline CO-RADS 3 & Indeterminate & $\begin{array}{l}\text { Unclear whether COVID-19 is } \\
\text { present }\end{array}$ \\
\hline CO-RADS 4 & High & $\begin{array}{l}\text { Abnormalities suspicious for } \\
\text { COVID-19 }\end{array}$ \\
\hline CO-RADS 5 & Very high & Typical COVID-19 \\
\hline CO-RADS 6 & PCR + & \\
\hline
\end{tabular}

This study aimed to point out the correlation between suspected COVID-19 patients with variable laboratory data as well as the CT findings during the second wave. Further correlation between positive PCR cases with laboratory and CT chest findings were done.

\section{PATIENTS AND METHODS}

295 patients (122 females and 173 males) with age ranging from 13 to 95 years and a mean of 58 years were admitted to our hospital with suspected COVID-19 pneumonia during the period from November 2020 to February 2021. They underwent multiple laboratory tests including $\mathrm{s}$. ferritin and $\mathrm{CBC}$ with differential count. Besides, non-contrast CT was done.

\section{CT imaging:}

CT scans were acquired for the 295 patients in the supine location on a Dual-source CT (Somatom Definition, Siemens Medical Solutions, Forchheim, Germany) with the subsequent parameters: $1.0-\mathrm{mm}$ section thickness, $5-\mathrm{mm}$ gap, $120 \mathrm{kV}$, and $150 \mathrm{~mA}$. The CT images were displayed with standard lung (window width, 1000 to $2000 \mathrm{HU}$; window level - 700 to $-500 \mathrm{HU}$ ) and mediastinal (window width 300 to $400 \mathrm{HU}$; window level 30 to $50 \mathrm{HU}$ ) settings.

The CT scans were assessed for the presence of ground-glass opacities and consolidation, as well as pleural effusion. 


\section{Ethical consent:}

An approval of the study was obtained from Ain Shams University Academic and Ethical committee. Every patient signed an informed written consent for acceptance of the study.

This work has been carried out in accordance with The Code of Ethics of the World Medical Association (Declaration of Helsinki) for studies involving humans.

\section{Statistical analysis}

IBM SPSS statistics (V. 26.0, IBM Corp., USA, 2019) was used for data analysis. Data were expressed as median and percentiles for quantitative nonparametric measures in addition to both number and percentage for categorized data. Comparison between two independent groups for non-parametric data was done using Wilcoxon Rank Sum test. P value $\leq 0.05$ was considered significant.

\section{RESULTS}

Among the 295 included patients, 122 were females $(41.4 \%)$ and 173 were males (58.6\%). The age distribution was ranging from 13 to 95 years with a mean of 58 years. As regards the CT findings, 198 (67.1\%) showed ground glassing while 32.9\% (97 cases) didn't. 36.9\% (109 cases) had consolidation in their CT while in 186 cases $(63.1 \%)$ there was no consolidation.

These lesions were bilateral in 181 cases about $63.3 \%$ and unilateral in $31.5 \%$ of the cases $(90$ patients) (Table 2) being single in 71 cases $(25.3 \%)$ and multiple in 200 cases $(71.5 \%)$ as shown in table (3).

Table (2): Bilaterality of lesions (GG and consolidations)

\begin{tabular}{|c|c|c|c|}
\hline 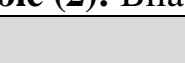 & & & Total \\
\hline \multirow[t]{6}{*}{ Bilaterality } & \multirow[t]{2}{*}{$\mathbf{B}$} & Count & 181 \\
\hline & & $\%$ & $63.3 \%$ \\
\hline & \multirow[t]{2}{*}{$\mathbf{N}$} & Count & 15 \\
\hline & & $\%$ & $5.2 \%$ \\
\hline & \multirow[t]{2}{*}{$\mathbf{U}$} & Count & 90 \\
\hline & & $\%$ & $31.5 \%$ \\
\hline \multirow{2}{*}{\multicolumn{2}{|c|}{ Total }} & Count & 286 \\
\hline & & $\%$ & $100.0 \%$ \\
\hline
\end{tabular}

Table (3): Number of lesions (GG and consolidations)

\begin{tabular}{|c|c|c|c|}
\hline 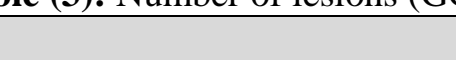 & & & Total \\
\hline \multirow[t]{6}{*}{ Multiplicity (Single /multiple) } & \multirow[t]{2}{*}{$\mathbf{M}$} & Count & 200 \\
\hline & & $\%$ & $71.2 \%$ \\
\hline & \multirow[t]{2}{*}{$\mathbf{N}$} & Count & 10 \\
\hline & & $\%$ & $3.5 \%$ \\
\hline & \multirow[t]{2}{*}{$\mathbf{S}$} & Count & 71 \\
\hline & & $\%$ & $25.3 \%$ \\
\hline \multirow{2}{*}{\multicolumn{2}{|c|}{ Total }} & Count & 281 \\
\hline & & $\%$ & $100.0 \%$ \\
\hline
\end{tabular}

202 cases $(68.7 \%)$ had pleural effusion, while in $31.3 \%$ (92 cases) there was no pleural effusion (Table 4)

Table (4): Presence of pleural effusion

\begin{tabular}{|c|c|c|c|}
\hline (2) & & & Total \\
\hline \multirow{4}{*}{ Pleural effusion } & \multirow{2}{*}{$\mathbf{N}$} & Count & 202 \\
\hline & & $\%$ & $68.7 \%$ \\
\hline & \multirow{2}{*}{$\mathbf{Y}$} & Count & 92 \\
\hline & & $\%$ & $31.3 \%$ \\
\hline \multirow{2}{*}{\multicolumn{2}{|c|}{ Total }} & Count & Count \\
\hline & & $\%$ & $\%$ \\
\hline
\end{tabular}

There was significant correlation between CRP, s. ferritin and d-dimer with presence of consolidation. Relation between ground glassing and age, lymphocytes, CRP \& ferritin were non-significant, however there was significant relation between neutrophils \& D-Dimer (DD). There was significant decrease of neutrophil count among positive in comparison with negative ground glassing and significant decrease of mean DD among positive in comparison with negative ground glassing (Table 5). 
Table (5): Relation between ground glassing and age, TLC, CRP \& ferritin, neutrophils \& D-Dimer

\begin{tabular}{|c|c|c|c|c|c|c|c|c|}
\hline 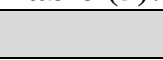 & Ground. glassing & $\mathbf{N}$ & Median & 25 Perc & 75 Perc & $\mathbf{Z}$ & p & Sig. \\
\hline \multirow{2}{*}{ Age } & $\mathrm{N}$ & 97 & 59 & 49.5 & 70 & \multirow{2}{*}{-1.619} & \multirow{2}{*}{0.105} & \multirow{2}{*}{ NS } \\
\hline & Y & 197 & 57 & 41 & 68 & & & \\
\hline \multirow{2}{*}{ TLC } & $\mathrm{N}$ & 82 & 8.6 & 6.2 & 14.4 & \multirow{2}{*}{-1.859} & \multirow{2}{*}{0.063} & \multirow{2}{*}{ NS } \\
\hline & $\mathrm{Y}$ & 162 & 7.85 & 5.3 & 11.3 & & & \\
\hline \multirow{2}{*}{$\mathrm{N}$} & $\mathrm{N}$ & 82 & 6467.3 & 3927.6 & 10980 & \multirow{2}{*}{-2.026} & \multirow{2}{*}{0.043} & \multirow{2}{*}{$S$} \\
\hline & $\mathrm{Y}$ & 162 & 5286.4 & 2910.95 & 8900.925 & & & \\
\hline \multirow{2}{*}{$\mathrm{L}$} & $\mathrm{N}$ & 82 & 1370.9 & 809.075 & 1987.725 & \multirow{2}{*}{-0.672} & \multirow{2}{*}{0.502} & \multirow{2}{*}{ NS } \\
\hline & $\bar{Y}$ & 162 & 1453.9 & 798.6 & 2120.5 & & & \\
\hline \multirow{2}{*}{ CRP } & $\mathrm{N}$ & 60 & 5.875 & 2.5025 & 12.1075 & \multirow{2}{*}{-1.723} & \multirow{2}{*}{0.085} & \multirow{2}{*}{ NS } \\
\hline & $\mathrm{Y}$ & 98 & 4.04 & 1.0825 & 10.6925 & & & \\
\hline \multirow{2}{*}{ Ferritin } & $\mathrm{N}$ & 17 & 558.3 & 173 & 933.55 & \multirow{2}{*}{-0.539} & \multirow{2}{*}{0.59} & \multirow{2}{*}{ NS } \\
\hline & $\mathrm{Y}$ & 54 & 344.95 & 136.675 & 719.6 & & & \\
\hline \multirow{2}{*}{$\begin{array}{l}\text { D-dimer } \\
\text { (DD) }\end{array}$} & $\mathrm{N}$ & 20 & 2.675 & 1.62 & 3.865 & \multirow{2}{*}{-2.364} & \multirow{2}{*}{0.018} & \multirow{2}{*}{ S } \\
\hline & $\mathrm{Y}$ & 63 & 1.24 & 0.41 & 2.5 & & & \\
\hline
\end{tabular}

There was significant decrease of neutrophil count among positive in comparison with negative ground glassing and significant decrease of mean DD among positive in comparison with negative ground glassing. Non-significant difference between CO-RADS categories \& age among all studied cases. PCR results were available in only 144 out of 295 patients (48.8\%). It was positive in 100 out of 144 patients (69.4\%) and negative in 44 patients (30.6\%) (Table 6).

Table (6): PCR status

\begin{tabular}{|c|c|c|c|}
\hline \multicolumn{2}{|c|}{} & Total \\
\hline \multirow{3}{*}{ PCR } & \multirow{2}{*}{ Neg } & Count & 44 \\
\cline { 2 - 4 } & & \% & $30.6 \%$ \\
\cline { 2 - 4 } & \multirow{2}{*}{ Pos } & Count & 100 \\
\cline { 2 - 4 } & & \% & Count \\
\hline \multicolumn{2}{|c|}{ Total } & Count & \% \\
\cline { 2 - 4 } & & \% within VAR00001 &
\end{tabular}

PCR was positive in $66 \%$ of cases with CO-RADS 5, 20\% in cases with CO-RADS 4, 8\% in CO-RADS 3 cases and only $4 \%$ and $2 \%$ in cases with CORADS 2 and 1 respectively. Highly significant relation between CO-RADS categories \& PCR among all studied cases (Table 7). As shown in the table (7), \% of positive PCR among CO-RADS categories 1,2 and 3 were lower than that of negative ( $2 \% \mathrm{Vs} 25 \%, 4 \% \mathrm{Vs} 18.2 \%$ and $8 \%$ Vs $20.5 \%)$, while they were higher than that of negative PCR among categories 4 and 5 (20\% Vs 18\% and 66\% Vs 5\%) i.e. increased $\%$ of positivity of PCR among higher categories of CO-RADS 4 and 5 Vs 1, 2 and 3.

Table (7): Relation between PCR \& CO-RADS

\begin{tabular}{|c|c|c|c|c|c|}
\hline & & & \multicolumn{2}{|c|}{ PCR } & \multirow[b]{2}{*}{ Total } \\
\hline & & & Negative & Positive & \\
\hline \multirow[t]{10}{*}{ CORADS } & \multirow[t]{2}{*}{1.00} & Count & 11 & 2 & 13 \\
\hline & & $\%$ & $25.0 \%$ & $2.0 \%$ & $9.0 \%$ \\
\hline & \multirow[t]{2}{*}{2.00} & Count & 8 & 4 & 12 \\
\hline & & $\%$ & $18.2 \%$ & $4.0 \%$ & $8.3 \%$ \\
\hline & \multirow[t]{2}{*}{3.00} & Count & 9 & 8 & 17 \\
\hline & & $\%$ & $20.5 \%$ & $8.0 \%$ & $11.8 \%$ \\
\hline & \multirow[t]{2}{*}{4.00} & Count & 8 & 20 & 28 \\
\hline & & $\%$ & $18.2 \%$ & $20.0 \%$ & $19.4 \%$ \\
\hline & \multirow[t]{2}{*}{5.00} & Count & 8 & 66 & 74 \\
\hline & & $\%$ & $18.2 \%$ & $66.0 \%$ & $51.4 \%$ \\
\hline \multirow{2}{*}{\multicolumn{2}{|c|}{ Total }} & Count & 44 & 100 & 144 \\
\hline & & $\%$ & $100.0 \%$ & $100.0 \%$ & $100.0 \%$ \\
\hline \multicolumn{6}{|c|}{ Chi-Square Tests } \\
\hline & & & & & $\mathbf{P}$ \\
\hline \multicolumn{3}{|c|}{ Pearson Chi-Square } & & $42^{\mathrm{a}}$ & .000 \\
\hline
\end{tabular}


(a)

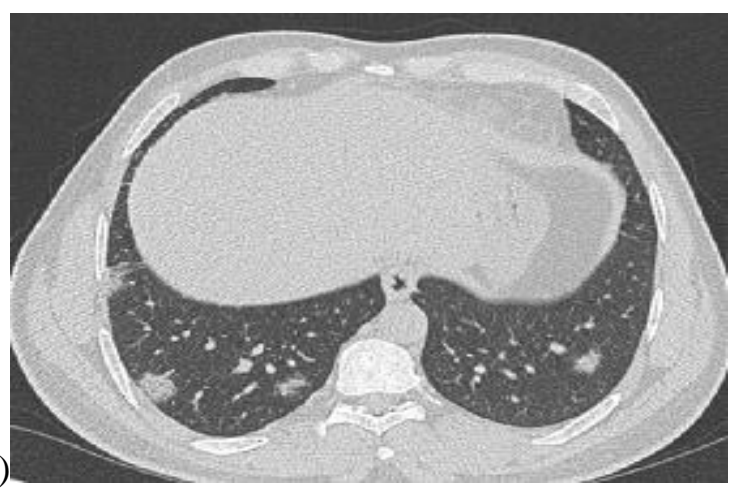

(b)

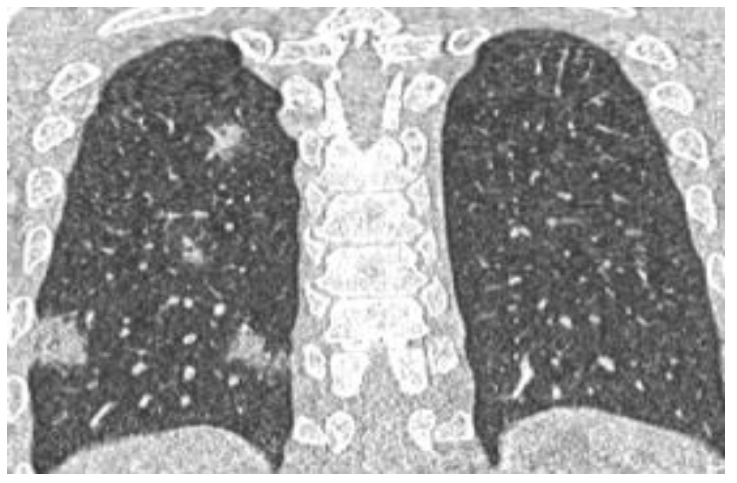

Fig. (1): A 34-year-old male patient with positive PCR, TLC 2.6, neutrophils 1518.4, Lymphocytes 785.2. Noncontrast CT (a) axial lung window and (b) coronal lung window revealed scattered bilateral peripheral ground glassing more evident at the lower lobes consistent with CO-RADS 5.

(a)
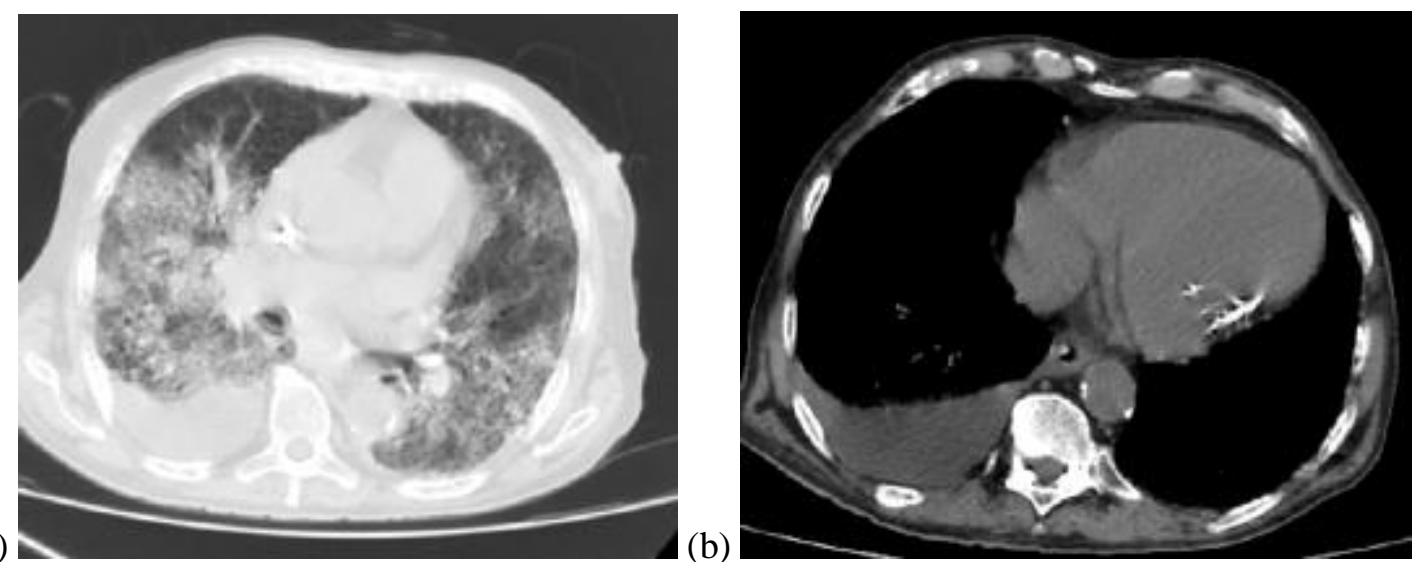

Fig. (2): A 79 year old male patient with positive PCR, with TLC 5.8, neutrophil count 5121.4, lymphocyte count 458.2, CRP 12, ferritin 1094 and D-dimer 2.6. Non contrast CT (a) axial lung window (b) axial mediastinal window revealed bilateral peripheral ground glassing with crazy paving consistent with CO-RADS 5. Moderate right sided pleural effusion was seen.

(a)

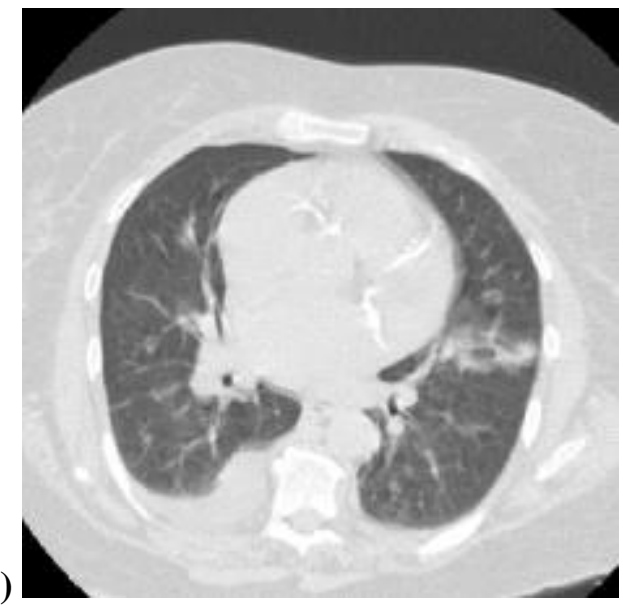

(b)

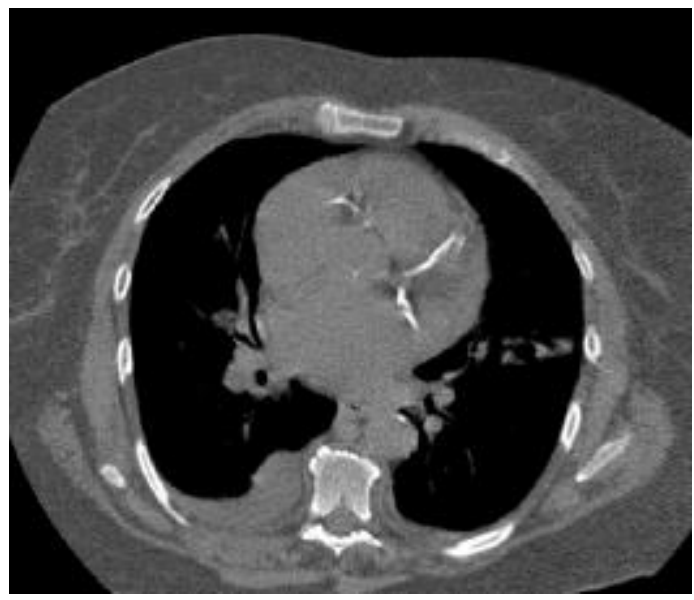

Fig. (3): 62 year old female patient with positive PCR, with TLC 6, neutrophil count 3450, lymphocyte count 1650, CRP 0.72, ferritin 27.8 and D-dimer 2.37. Non contrast CT (a) axial lung window and (b) axial mediastinal window revealed a single lingular peripheral patch of ground glassing consistent with CO-RADS 3. Moderate right sided pleural effusion was seen. 
(a)

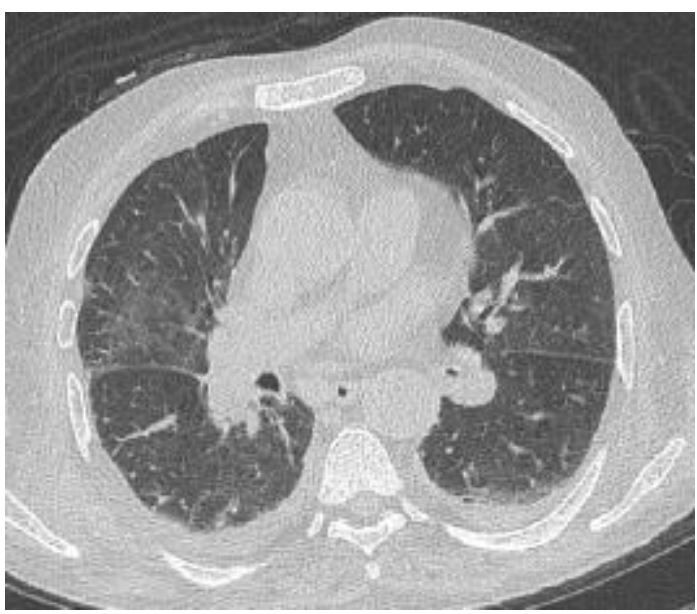

(b)

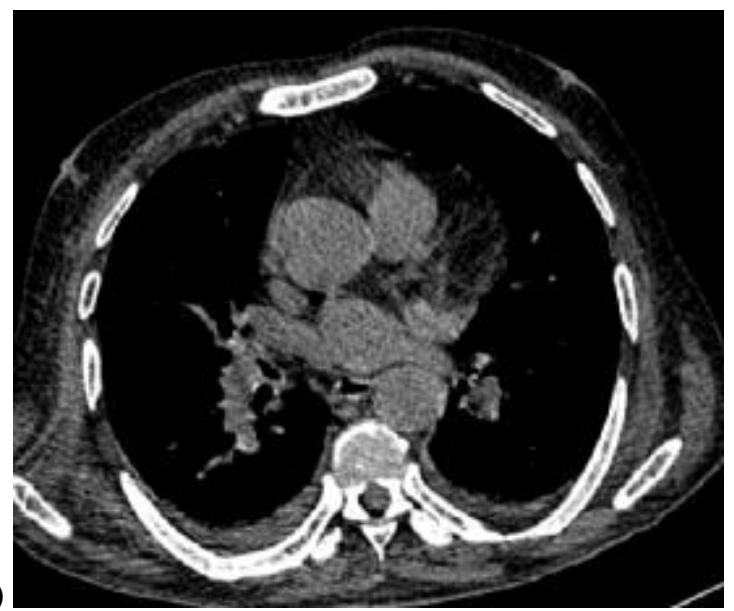

Fig. (4): A 83 year old male patient complaining of fever with negative PCR, with TLC 18.4, neutrophil count 16228.8, lymphocyte count 1269.6, CRP 18.89, ferritin 268.2 and D-dimer 1.57. Non contrast CT revealed mild bilateral pleural effusion with no ground glassing or consolidations, consistent with CORADS 2.

\section{DISCUSSION}

As regards the CT findings, our study showed that $198(67.1 \%)$ showed ground glassing, this is keeping with Fu et al. ${ }^{\left({ }^{6}\right)}$ where forty of fifty-six patients $(83.6 \%)$ had two or more opacities in the lung. Our results are also matching with Vancheri et al. ${ }^{(7)}$ where ground glass opacity (GGO), either alone or accompanied with other changes was noted in 124/180 patients $(68.8 \%)$. This is also matching with a study by Wu et al. ${ }^{(8)}$, which revealed that the most frequent CT abnormalities observed were ground glass opacity (73/80 cases, $91 \%)$ followed by consolidation $(50 / 80$ cases, 63\%). In another study by Guan et al. ${ }^{(9)}, 53$ patients with COVID-19 were enrolled and GGO was reported in all of them (100\%). Ng et al. ${ }^{(\mathbf{1 0})}$ stated that GGO occurred in $86 \%$ of his patients.

Thirty four of 296 patients $(11.4 \%)$ had both ground glassing and consolidative patches in our study. Unlike Fu et $\boldsymbol{a l} .^{(6)}$ where twenty-nine patients $(52.7 \%)$ had ground-glass and consolidative opacities. This is keeping with Altmayer $\boldsymbol{e t}$ al. ${ }^{(11)}$ who revealed that GGO was the commonest CT finding, being present in up to 0.92 (95\% CI, 0.90-0.97) of COVID19 and 0.80 (95\% CI, 0.74-0.85) of non-COVID, followed by consolidation (COVID-19, 0.50; 95\% CI 0.33-0.66; non-COVID, 0.69, 95\% CI 0.61-0.77).

In our study, 36.9\% (109 cases) had consolidation either alone or with GGO in their CT. This is matching with Vancheri $\boldsymbol{e t}$ al. ${ }^{(7)}$ results, which revealed that consolidation accompanied with other findings was detected in $71 / 180$ patients $(39.4 \%)$. A meta-analysis study done by Bao et al. ${ }^{(12)}$ revealed that the typical CT findings were GGO (83.31\%) followed by GGO associated with consolidation $(58.42 \%)$ where the most anatomical distributions were bilateral lung infection (78.2\%) and peripheral distribution $(76.95 \%)$.

74 of our 295 cases (25\%) had only consolidation without ground glassing, while in a study by Fu et al. (6) eight patients (14.5\%) had consolidation solely. According to Vancheri et al. ${ }^{(7)}$
GGO and reticular alteration were significantly more frequent than consolidation ( $\mathrm{p}<0.01$ in both cases). This is matching with our results, which showed that 198 of our patient's CT showed GGO $(67.1 \%)$, while consolidation patches were detected in 109 patients $(36.9 \%)$. This also is keeping with a study done by Sui et al. ${ }^{(13)}$, which showed that the predominant pattern was ground-glass opacities, while consolidation became the second most common pattern. 181 patients $(63.3 \%)$ showed bilateral lung involvement, which is matching with Sui et al. ${ }^{(13)}$.

The PCR results were available in only 144 of our cases. Of these 100 cases $(69.4 \%)$ were positive while the remaining 44 patients had negative PCR results. Of the 100 patients with positive PCR results, 67 of them showed typical CT findings for COVID according to the RSNA criteria (67\%) (Figures 1 and $2)$ and only two cases (2\%) were completely free i.e. normal by RSNA criteria. The rest of the cases showed variable findings (either atypical or indeterminate) (Fig. 3). Of the 1014 patients, 601 had positive RTPCR results and 413 had negative RT-PCR results, for a positive rate of 59\%. Of the 601 patients with positive RT-PCR results, 580 (97\%) had positive chest CT scans. Of the 413 patients with negative RT-PCR results, 308 (75\%) had positive chest CT scans (Fig. 4). This is also in agreement with Ai et al. ${ }^{(14)}$ where out of 1014 patients, 888 (88\%; 95\% CI: 86\%, 90\%) had positive chest CT. The main chest CT findings were ground-glass opacity (409 of 888 patients (46\%)) and consolidations (447 of 888 patients (50\%)). Most patients $(801$ of $888(90 \%))$ had bilateral chest CT findings.

In our study, 202 cases $(68.7 \%)$ had pleural effusion, which is contrary to a study by Vancheri $\boldsymbol{e t}$ al. ${ }^{(7)}$ who reported that pleural effusion was observed in $12 / 180$ patients $(6.6 \%)$, being unilateral in all of them. This is also matching the meta-analysis study done by Bao et al. ${ }^{(12)}$ were pleural effusion was found in $5.88 \%$. This also matches with Altmayer et al. ${ }^{(11)}$ 
who reported that pleural effusion was rare in COVID19 (0.03; 95\% CI 0.01-0.04).

Highly significant relation between CO-RADS categories \& PCR was found among all our cases with increased percentage of positivity of PCR among higher categories of CORADS 4 and 5 Vs 1, 2 and 3 as described in table (6). In a meta-analysis study by Kwee et al. ${ }^{(5)}$, providing pooled data regarding the frequency of proved cases with COVID-19 infection with the different categories of CO-RADS and the RSNA classification systems, revealed that with the higher CO-RADS and RSNA categories, the frequency of proved cases of COVID-19 increased.

PCR was positive in $66 \%$ of cases with CORADS 5, 20\% in cases with CORADS $4,8 \%$ in CORADS 3 cases and only $4 \%$ and $2 \%$ in cases with CORADS 2 and 1 respectively. This is keeping with a meta-analysis study done by Kwee et al. ${ }^{(5)}$ who concluded that in CO-RADS 5, the prevalence of COVID-19 was $89.6 \%$.

In our study, 75 patients showed typical RSNA, 70 of them were positive PCR for COVID-19 pneumonia (93.3\%). This is keeping with Kwee et al. ${ }^{(5)}$ results in that in cases with typical RSNA category, the frequency of COVID-19 was $92.5 \%$.

Our study revealed that there was a significant relation between neutrophils \& D-Dimer with GGO on CT. There was significant decrease of neutrophil count among positive in comparison with negative ground glassing and significant decrease of mean DD among positive in comparison with negative ground glassing. This is not matching with both Wang ${ }^{(15)}$ and Rahman et al. ${ }^{(16)}$ where they both suggested that the CRP levels were positively correlated with lung lesions and could reflect disease severity. Rahman et al. (16) also reported that the d-dimer and ferritin showed good indicative value to evaluate the severity of COVID-19.

By using standardized diagnostic criteria including CO-RADS or the RSNA classification, a higher percentage of non-COVID-19 patients with other lung pathologies due to other lung diseases will be justly reported as COVID-19 free with an alternate lung disease ${ }^{(\mathbf{1 1})}$.

Our study had some limitations: First, that not all our cases had PCR results. Furthermore, the relatively low number of patients as the hospital is a tertiary university center not COVID center.

\section{CONCLUSION}

In conclusion, during the second wave, the CT findings were similar to wave one with PCR proved COVID-19 cases more frequent with higher CORADS and RSNA categories. The common CT features of COVID-19 pneumonia were multiple bilateral ground glassing, followed by consolidation. Both CO-RADS and RSNA classification systems have strong positive correlation with PCR.

Financial support and sponsorship: Nil.

Conflict of interest: Nil.

\section{REFERENCES}

1. Rubin G, Ryerson C, Haramati L et al. (2020): The Role of Chest Imaging in Patient Management During the COVID-19 Pandemic: A Multinational Consensus Statement From the Fleischner Society. Chest, 158 (1): 106-116.

2. Bernheim A, Mei X, Huang M et al. (2020): Chest CT Findings in Coronavirus Disease-19 (COVID-19): Relationship to Duration of Infection. Radiology, 295 (3):200463.

3. Prokop $M$, van Everdingen $W$, van Rees Vellinga $\mathbf{T}$ et al. (2020): CO-RADS: A Categorical CT Assessment Scheme for Patients Suspected of Having COVID-19Definition and Evaluation. Radiology, 296 (2): 1-5.

4. Francone M, Iafrate F, Masci G et al. (2020): Chest CT score in COVID-19 patients: correlation with disease severity and short-term prognosis. Eur Radiol., 20: 1-10.

5. Kwee R, Adams H, Kwee $\mathbf{T}$ (2021): Diagnostic Performance of CO-RADS and the RSNA Classification System in Evaluating COVID-19 at Chest CT: A MetaAnalysis. Radiology: Cardiothoracic Imaging, 3 (1): e200510.

6. Fu F, Lou J, Xi D et al. (2020): Chest computed tomography findings of coronavirus disease 2019 (COVID-19) pneumonia. Eur Radiol., 30 (10): 54895498.

7. Vancheri S, Savietto G, Ballati F et al. (2020): Radiographic findings in 240 patients with COVID-19 pneumonia: time-dependence after the onset of symptoms. Eur Radiol., 30: 6161-6169.

8. Wu J, Wu X, Li C (2020): Chest CT Findings in Patients with Coronavirus Disease 2019 and Its Relationship with Clinical Features. Invest Radiol., 55 (5): 257-261.

9. Guan C, Lv Z, Yan S et al. (2020): Imaging features of coronavirus disease 2019 (COVID-19): evaluation on thin-section CT. Acad Radiol., 27 (5): 609-613.

10. Ng M, Lee E, Yang J et al. (2020): Imaging profile of the COVID-19 infection: radiologic findings and literature review. Radio Cardiothorac Imaging, 2: 200034.

11. Altmayer S, Zanon M, Pacini G et al. (2020): Comparison of the computed tomography findings in COVID-19 and other viral pneumonia in immunocompetent adults: a systematic review and metaanalysis. European Radiology, 30 (12): 6485-6496.

12. Bao $C$, Liu $X$, Zhang $H$ et al. (2020): Coronavirus Disease 2019 (COVID-19) CT Findings: A Systematic Review and Meta-analysis. J Am Coll Radiol., 17: 701709.

13. Sui H, Han $X$, Jiang $N$ et al. (2020): Radiological findings from 81 patients with COVID-19 pneumonia in Wuhan, China: a descriptive study. Lancet Infect Dis., 20: 425-34.

14. Ai T, Yang Z, Hou H et al. (2020): Correlation of Chest CT and RT-PCR Testing for Coronavirus Disease 2019 (COVID-19) in China: A Report of 1014 Cases. Radiology, 296: 32-40.

15. Wang $\mathbf{L}$ (2020): C-reactive protein levels in the early stage of COVID-19. Med Mal Infect., 50 (4): 332-334.

16. Rahman M, Shanjana Y, Tushar M et al. (2021): Hematological abnormalities and co-morbidities are associated with COVID-19 severity among hospitalized patients: Experience from Bangladesh. PLoS One, 16 (7): 0255379 . 